Western University

Scholarship@Western

Communication Sciences and Disorders

Publications

Communication Sciences and Disorders School

$1-1-2018$

\title{
Maturation in auditory event-related potentials explains variation in language ability in children
}

\author{
Elaine Y.L. Kwok \\ The University of Western Ontario \\ Marc F. Joanisse \\ The University of Western Ontario \\ Lisa M.D. Archibald \\ The University of Western Ontario \\ Margot E. Stothers \\ The University of Western Ontario \\ Heather M. Brown \\ The University of Western Ontario
}

See next page for additional authors

Follow this and additional works at: https://ir.lib.uwo.ca/scsdpub

Citation of this paper:

Kwok, Elaine Y.L.; Joanisse, Marc F.; Archibald, Lisa M.D.; Stothers, Margot E.; Brown, Heather M.; and Oram Cardy, Janis, "Maturation in auditory event-related potentials explains variation in language ability in children" (2018). Communication Sciences and Disorders Publications. 19.

https://ir.lib.uwo.ca/scsdpub/19 


\section{Authors}

Elaine Y.L. Kwok, Marc F. Joanisse, Lisa M.D. Archibald, Margot E. Stothers, Heather M. Brown, and Janis Oram Cardy 
Western University Scholarship@Western

$1-1-2018$

\section{Maturation in auditory event-related potentials explains variation in language ability in children.}

Elaine Yuen Ling Kwok

Health and Rehabilitation Science, Western University, ekwok5@uwo.ca

Marc F Joanisse

Lisa Archibald

Western University, larchiba@uwo.ca

Margot E. Stothers

mstothe2@uwo.ca

Heather M Brown

See next page for additional authors

Follow this and additional works at: https://ir.lib.uwo.ca/hrspub

Part of the Developmental Neuroscience Commons

\section{Citation of this paper:}

Kwok, E. Y., Joanisse, M. F., Archibald, L. M., Stothers, M. E., Brown, H. M., \& Oram Cardy, J. (2018). Maturation in auditory event-related potentials explains variation in language ability in children. European Journal of Neuroscience, 47(1), 69-76. 
Authors

Elaine Yuen Ling Kwok, Marc F Joanisse, Lisa Archibald, Margot E. Stothers, Heather M Brown, and Janis Oram Cardy 
Maturation in Auditory Event-Related Potentials Explains Variation in Language Ability in Children

Elaine Y. L. Kwok ${ }^{1}$, Marc F. Joanisse ${ }^{3,4}$, Lisa M. D. Archibald ${ }^{13}$, Margot E. Stothers ${ }^{2}$, Heather M. Brown $^{2}, \&$ Janis Oram Cardy'

'School of Communication Sciences and Disorders, ${ }^{2}$ Health and Rehabilitation Sciences, and ${ }^{3}$ Department of Psychology ${ }^{4}$ Brain and Mind Institute

The University of Western Ontario

Author Note:

Heather Brown is now at the Department of Educational Psychology, University of Alberta

Contact Information:

Dr. Janis Oram Cardy

School of Communication Sciences and Disorders

Elborn College, The University of Western Ontario

London, Ontario, N6G 1H1

Tel: 15196612111 x88000; Fax: 15198502369

Email: janis.cardy@uwo.ca

Keywords: EEG; auditory cortex; language development; neurodevelopment; maturation 


\begin{abstract}
Processing of auditory information in the cortex continues to develop into later childhood and adolescence. Recent research has indicated that intraclass correlation (ICC) is the best method for capturing maturation in auditory event-related potentials (AEPs) of school-age children. However, the sensitivity of the ICC approach in discerning AEP changes in children has not been consistently demonstrated and positive results have not been replicated. We attempted this replication and further explored whether AEP maturation estimated using the ICC approach predicts cognitive and linguistic abilities in addition to chronological age. We measured AEPs in response to simple tones in groups of $7,8,9$, and 10 year olds with typical development $(N=$ 67), and used ICC to estimate the age equivalent of each child's AEP (AEP-age). Results indicated that ICC differentiated 7-8- from 9-10-year-old children, and that AEP-age predicted both chronological age and significant, unique variance in language ability, but not in nonverbal IQ. Our findings support the view that auditory organization in children reflects both general developmental maturation and more specific development of language skills, and support the future use of AEP-age to identify and understand individual differences in brain maturation in typically developing and clinical populations.
\end{abstract}




\section{Introduction}

Studies examining cortical auditory evoked potentials (AEPs) have found that processing of auditory stimuli does not become fully mature until late adolescence or adulthood (Bender $e t$ al., 2006; Bishop et al., 2011; Fox et al., 2010; Pang \& Taylor, 2000; Ponton et al., 2000; Sussman et al., 2008). Intraclass correlation (ICC) has been suggested to be the best analysis method for estimating maturational level of AEPs (Bishop et al., 2011). However, the sensitivity and reliability of this analysis in school-age children has not been consistently demonstrated. Moreover, while research using traditional electroencephalography (EEG) analysis techniques (such as component-based analysis) has suggested a relation between AEP maturation and language development, it remains unclear whether AEP maturation indexed via ICC analysis is also related to language development. This study aimed to further examine the utility of an ICCderived AEP maturity index by examining its sensitivity to change in AEPs in school-age children and its ability to capture relations between auditory maturation and oral language.

A number of studies have characterized developmental changes in auditory processing by examining AEPs. These automatic neural responses to auditory stimuli, observed in discrete waveforms extracted using EEG, are of particular interest because they can be measured with little cognitive demand on participants. The most distinct complex of AEPs appears from approximately 50 to $250 \mathrm{~ms}$ after the onset of an auditory stimulus, and includes several predictable waveform components: P1, N1, P2, and N2 (Ponton et al., 2000). These components have been shown to change both in latency and amplitude as children mature (Bender et al., 2006; Fox et al., 2010; Ponton et al., 2000; Sussman et al., 2008). Although EEG analysis methodologies are available for quantifying the amplitude or latency of specific components, these methodologies can provide spurious estimation when the component under investigation is not present in the EEG waveform (McArthur \& Bishop, 2004). This consideration is relevant when studying developmental populations because some AEP components (e.g., N1) only 
emerge in late childhood (Ponton et al., 2000), can be delayed or absent in neurodevelopmental disorders (Edgar et al., 2014; Shafer et al., 2011), and can be highly influenced by the rate of stimulus presentation (Čeponiene et al., 1998).

Bishop et al. (2011) compared various EEG analysis approaches for capturing AEP maturation in children with typical development aged 7, 9, and 11 years. Compared to conventional analyses of peak amplitude and latency, independent component source localization, and time-frequency analysis, ICC was proposed to provide a superior estimate of auditory maturity. In their study, three grand averages were generated by averaging the responses of participants in each of the three age groups. Then, individual participant's AEPs were compared to each of the three grand averages using ICC. The higher the similarity between two AEP waveforms, the higher the resultant ICC value. The highest ICC between the individual AEPs and one of the age-related grand averages was taken to reflect the participant's auditory ERP age equivalent, or AEP-age. Bishop et al. found that while all of their EEG analysis methodologies demonstrated age-related AEP maturation, ICC analysis alone allowed for an estimate of the degree of maturity of each participant's AEP, that is, a "brain age." Such a maturational index is of interest because it has the potential to be used for the study of children from a broad range of ages and developmental abilities in future research and clinical applications, given its ability to be generated in response to simple, nonlinguistic stimuli in short, passive paradigms, and its lack of reliance on the presence of individual components in the AEP. Furthermore, because ICC calculation can take into account both amplitude and latency of multiple AEP components, it provides a global index that reduces the need for multiple comparisons and avoids the associated risk of inflated $p$-values (Bishop \& McArthur, 2005).

Although Bishop et al. (2011) found that ICC calculation was sensitive to typical developmental changes in AEPs from 7 to 9 to 11 years, this has not been consistently 
demonstrated. In an earlier study, Bishop et al. (2007) examined the use of ICC for characterizing maturational changes of AEPs from childhood to adulthood. They found that an ICC-derived age estimate could only discern AEP maturation across three broad age bands: 5-12 years, 13-16 years, and adulthood. In contrast to the 2011 findings, ICC calculation in the 2007 study failed to discern developmental changes in AEPs during the childhood years. The authors suggested that greater sensitivity of the ICC approach in the 2011 study was attributable to (a) a larger sample size, (b) the use of a longer interstimulus interval that increased the likelihood of eliciting a later-emerging N1 component, and (c) the use of a cross-sequential sample that reduced between-subject variance and isolated age-related maturation. These inconsistent findings motivated the first goal of our study, namely, to clarify the utility of ICC in discerning year-by-year maturational changes in AEPs in children from 7 to 10 years of age. Confirming its utility would be a necessary first step to establishing ICC as a reliable alternative method of AEP analysis in developmental populations.

Should an ICC-derived index prove to be useful in capturing maturational change in AEPs, it would have the potential to be used to study the relation between auditory cortical maturation and broader functional abilities in children with typical and atypical development. Indeed, studies of children with typical and impaired language development have pointed to a relation between maturation in the auditory cortex and language ability. For example, the amplitude or latency of AEP components in early childhood predicted later language and reading abilities (Benasich et al., 2006; Espy et al., 2004; Molfese et al., 1999, 2000). Because no research to date has established this link using an ICC-based AEP age equivalent, the second goal of our study was to determine if such a relation could be observed using the ICC approach. We further asked whether such a relation is domain-specific (i.e., restricted to the language domain) or related to global cognitive ability (i.e., including but not restricted to language). In 
the latter case, we would expect to find similar relations between AEP maturation and language ability, and AEP maturation and nonverbal cognitive abilities.

We examined AEPs evoked by single tones in a cross-sectional sample of children with typical development aged 7, 8, 9, and 10 years using the ICC analytic approach. Our purposes were to (a) confirm that ICC is a reliable method for capturing developmental changes in AEPs in this age range by attempting to replicate the ICC results of Bishop et al. (2011), (b) explore whether age-related change is more evident in particular channels from different scalp regions, and (c) examine the relation of AEP maturation estimated using ICC to factors other than chronological age, namely, oral language ability and nonverbal IQ.

\section{Materials and methods}

Western's Health Sciences Research Ethics Board approved the study, which was undertaken with the understanding and written consent of each child's parent and verbal assent of each child, and which conforms with the World Medical Association Declaration of Helsinki. Participants

Sixty-seven children aged 7 to 10 years participated in this study $(n=31$ Male, $n=36$ Female). All children were primary English speakers with no neurological, hearing, or visual impairment by parent report. Participants completed the Wechsler Abbreviated Intelligence Scale (WASI; Wechsler, 1999) and Clinical Evaluation of Language Fundamentals-4 (CELF-4; Semel et al., 2003). To be included in the study, children must have received WASI Full Scale IQ, Verbal IQ, Performance IQ, and CELF-4 Core Language standard scores at or above 85, that is, no more than one standard deviation below the mean.

Most of the participants were recruited from a large-scale investigation of the language, reading, and arithmetic skills of school-age children. Children completed standardized testing that included the WASI and CELF-4, as well as tests of memory and academic achievement 
reported previously (see Archibald et al., 2013). Ten participants were recruited from personal contacts, and completed the standardized tests in our university lab.

Procedure

Each child completed testing in a single visit to our university lab, during which auditory ERPs were recorded. To encourage an alert-but-still state during recordings, children watched a movie without sound. Auditory ERPs were evoked with the presentation of $490 \mathrm{~Hz}$ pure tones. Tones were $50 \mathrm{~ms}$ in duration with onset/offset ramps of $10 \mathrm{~ms}$, digitized at a $41.1 \mathrm{kHz}$ sampling rate using Praat software (Boersma \& Weenink, 2011). Responses to 225 tone repetitions were recorded over about 5 minutes, with interstimulus interval randomly jittered between 1000$1400 \mathrm{~ms}$ in $100 \mathrm{~ms}$ increments.

\section{EEG Acquisition and Processing}

Event-related encephalograms were recorded at $250 \mathrm{~Hz}$ using a 128-channel HydroCel Geodesic Sensor Nets (Electrical Geodesics Inc., Eugene, OR, USA). Data were recorded with an online bandpass filter of $0.1-100 \mathrm{~Hz}$ with $60 \mathrm{~Hz}$ notch filter, and were later filtered offline using 2-30 Hz finite impulse response (FIR) filter. Channels with impedances higher than $75 \mathrm{k} \Omega$ were excluded from subsequent analyses. Participants sat in a comfortable chair while auditory stimuli were presented binaurally via a digital-to-analogue conversion device (UA-25, Edirol Inc., Japan), an amplification system (Series III, Tucker Davis Technologies Inc., Florida, USA), and insert earphones (ER3A, Etymotic Research, Illinois, USA). Stimulus presentation was controlled using E-Prime software (Psychology Software Tools Inc., Pittsburgh, PA). Auditory stimuli were presented at $50 \mathrm{~dB}$ above each child's hearing threshold (i.e., dB SL), which was assessed using a 2-up/1-down procedure. Average referenced data were segmented into $1200 \mathrm{~ms}$ epochs (including $200 \mathrm{~ms}$ pre-stimulus baseline) that were time-locked to the onset of the tone. AEPs were computed by averaging artifact-free (movement artifacts, eye blinks, eye movements) segments. In addition, four grand average waveforms were created, each using 
AEPs from all children of the same age ( 7 years, $n=15 ; 8$ years, $n=16$; 9 years, $n=22 ; 10$ years, $n=14)$. On average, 110 artifact-free trials were available per participant. There was no difference in the number of accepted trials across age groups, $p=0.15$.

Data Analysis

Following the approach of Bishop et al. (2011), the Fisher-transformed intraclass correlation (ICC) statistic was used to measure the similarity of each child's waveform from 0$500 \mathrm{~ms}$ to grand average waveforms from each of the four age groups at nine electrodes (F3, Fz, $\mathrm{F} 4, \mathrm{C} 3, \mathrm{Cz}, \mathrm{C} 4, \mathrm{~T} 7, \mathrm{Pz}, \mathrm{T} 8$ ). These grand average waveforms are presented in Figure 1. For each $500 \mathrm{~ms}$ AEP waveform entered into ICC calculation, a total of 125 data points were collected (500ms $* 250 \mathrm{~Hz}$ sampling rate). It can be helpful to conceptualize the ICC calculation within the context of inter-rater reliability, a common application of ICC analysis. The 125 data points from each of the two AEP waveforms under comparison (i.e., one from an individual child and one that is the grand average for a specific age group) are akin to arrays of ratings provided by 125 pairs of raters. The ICC value was calculated between the two arrays of ratings using the

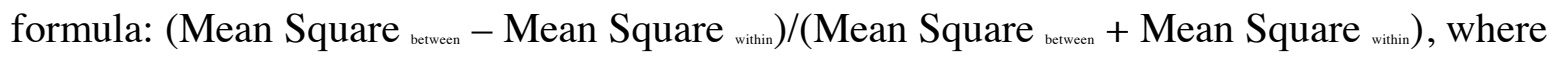

1. Mean Square semerea $=\left\{\left[\sum \mathrm{X}^{2}+\sum \mathrm{Y}^{2}+2 * \sum(\mathrm{X} . \mathrm{Y})\right] / 2-\left(\sum \mathrm{X}+\sum \mathrm{Y}\right)^{2} / 2 \mathrm{~N}\right\} /(N-1)$,

2. Mean Square ${ }_{\text {witini }}=\left[0.5 *\left(\sum \mathrm{X}^{2}+\sum \mathrm{Y}^{2}\right)-\sum(\mathrm{X} . \mathrm{Y})\right] / N$,

3. $\quad N=$ number of EEG data points entered into the ICC calculation, and

4. $\quad \mathrm{X}, \mathrm{Y}=$ the two AEP waveforms under comparison.

The resulting ICC provided a reliability estimate between the two arrays of ratings, and in this case, reflected the similarity of two AEP waveforms under comparison. This calculation was repeated for each participant at each electrode. The highest ICC across the four grand-average comparisons was taken to be that child's age-equivalent (namely, 7, 8, 9, or 10 years) for that electrode. Note that to avoid inflation of the ICC value, individual participant's waveforms were taken out of the corresponding grand average for their own chronological age group prior to ICC 
calculation. Figure 2 presents the AEP waveforms at channel $\mathrm{Cz}$ from one participant as compared to the four grand averages at $\mathrm{Cz}$ to illustrate this step of the analysis.

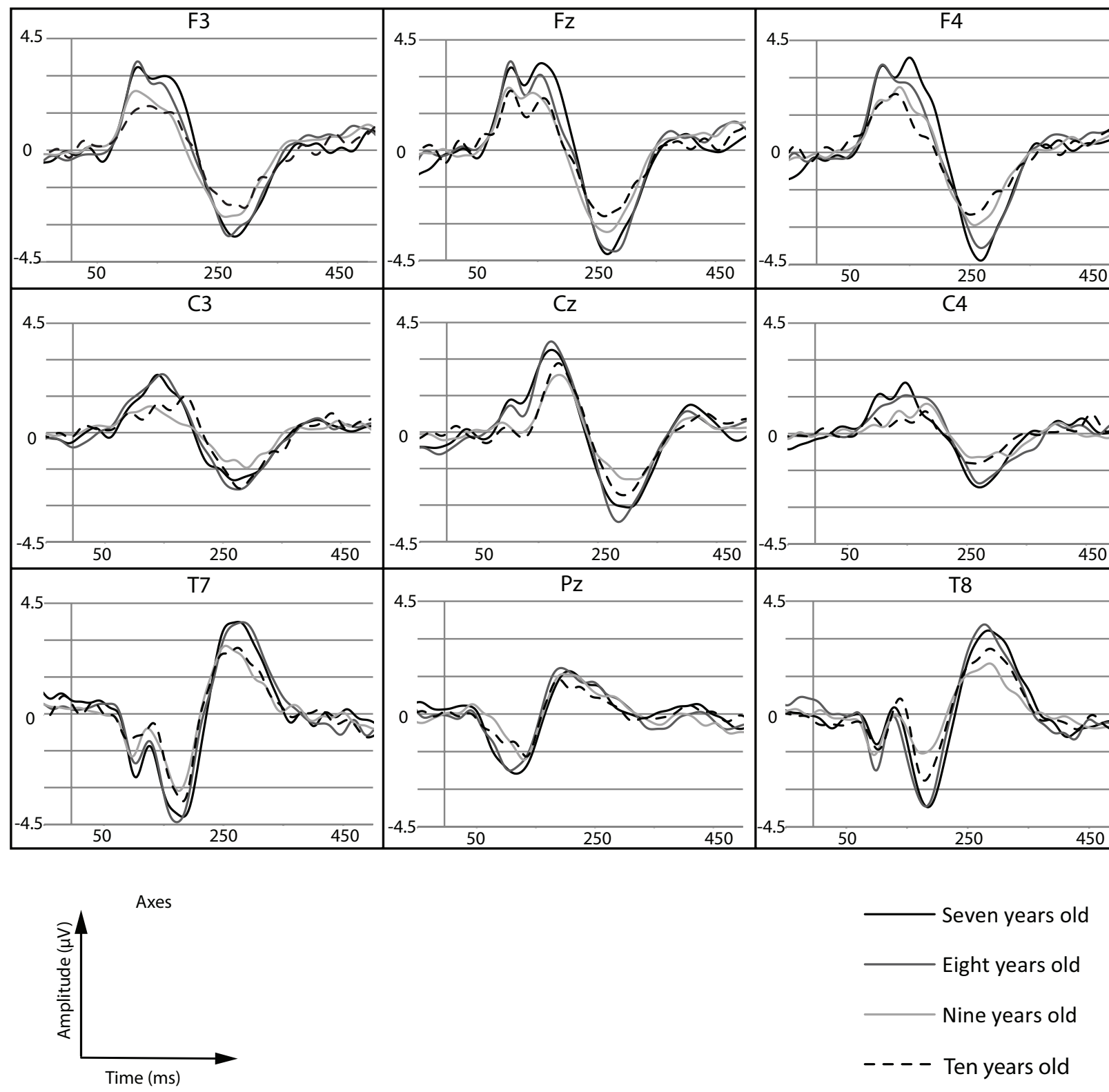

Figure 1. The grand average AEPs of the four chronological age groups at nine selected channels are overlaid to illustrate developmental change (x-axis, time in ms, y-axis amplitude $\mu \mathrm{V})$. The AEP-age of children aged 7-8 years significantly differed from those aged 9-10 years. 


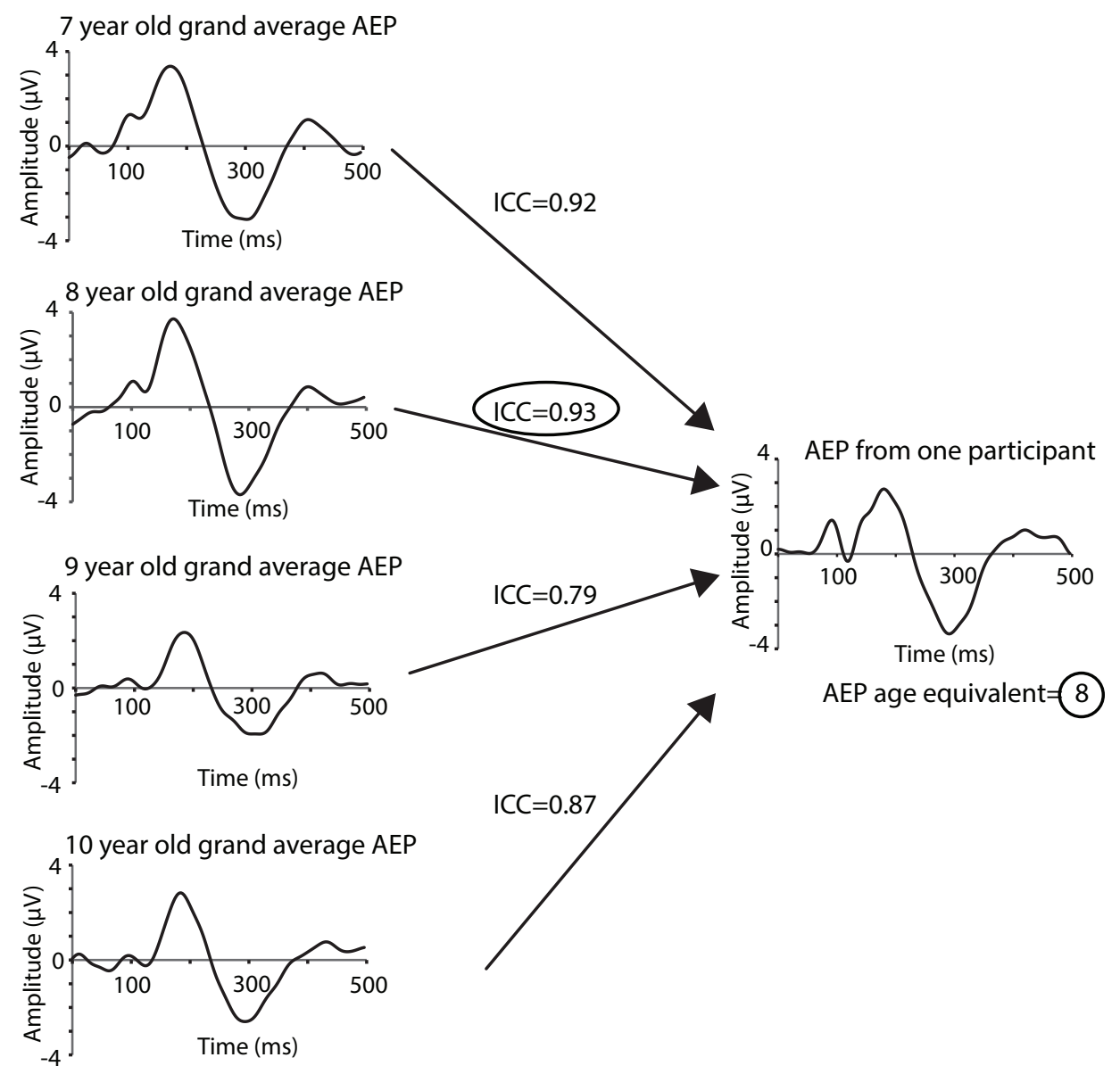

Figure 2. To determine the age equivalent of a child's AEP, the grand average AEP waveforms at a single channel for each of the four normative age groups (left) were compared to the AEP from the participant (right) using ICC. The comparison that resulted in the highest ICC value (in this case, the 8-year-old grand average) was taken as the age-equivalent of that AEP waveform for that channel. After age-equivalents were obtained from all selected channels, they were averaged to form AEP-age, an index of the overall maturity of the participant's cortical response to the tone. 
The resultant nine age-equivalents (from the 9 channels) were averaged together to generate the child's overall auditory ERP age (AEP-age). Potential mean differences in AEPage, CELF-4 Core Language, and WASI Performance IQ scores by chronological age group were explored with one-way analyses of variance. Note that language and nonverbal cognition are highly correlated with age. Using standardized, rather than raw, Core Language and Performance IQ scores in our analyses allowed us to examine the strength of each child's abilities in these areas relative to same-age peers, that is, controlling for age.

Bishop et al. (2007) demonstrated that differences in ICC values across age groups were smaller at temporal and parietal sites than for other electrodes (e.g., at central and frontal sites). This finding is consistent with other AEP literature showing the most robust maturational changes at frontal and central channels (Bishop et al., 2011; Ponton et al., 2000, 2002). Therefore, ICC analysis may not be a sensitive measure of AEP maturation across all channels. Given this, we also conducted a data-driven analysis in which we estimated AEP-age relying only on those channels showing close correspondence with chronological age. To identify electrodes that best reflected age-related changes, one-tailed correlational analyses with Bonferroni correction $(\alpha=0.05 / 9)$ were conducted between the age-equivalents of the nine selected channels and the chronological age of each participant. A refined AEP-age was calculated using only those channels that were significantly correlated with chronological age.

Finally, to explore the ability of AEP-age to predict age, language, and nonverbal IQ, regression analyses were conducted. First, to replicate the approach taken by Bishop et al . (2011), AEP-age was used as a predictor of chronological age. A separate regression analysis was conducted to examine whether language and cognitive abilities can be predicted by AEPage. To predict variance in CELF-4 Core Language and WASI Performance IQ scores, a hierarchical regression analysis included chronological age in step 1 and AEP-age in step 2 in order to explore whether additional variance could be explained above and beyond the effect of 
age-related maturation. Tolerance and Variance-inflation factor (VIF) values were calculated to ensure low multi-collinearity of the predictors (Tolerance $>.83$, VIF $<1.21$ ).

\section{Results}

As observed in the grand average AEP waveforms for each age group presented in Figure 1 , voltage, particularly at frontal and central channels, decreases with age. Overall, frontal and central channels recorded the greatest activation during the time window of interest in this study.

One-way analysis of variance revealed that the groups differed significantly in their AEP-age averaged across nine channels, $F(3,66)=7.88, \mathrm{p}<0.001, \eta^{2}=0.27$ (see Table 1). Post-hoc comparisons using Tukey's b revealed that both the 9- and 10-year-old groups had a significantly higher AEP-age than the 7-and 8-year-old groups. No difference in AEP-age was found between the 7- and 8-year-old or between the 9- and 10-year-old groups. Note that none of the age groups differed in CELF-4 Core Language, $F(3,66)=0.54, p=0.66, \eta_{p}=0.02$, or WASI Performance IQ scores, $F(3,66)=0.75, p=0.53, \eta^{2}=0.04$ (see Table 1$)$.

Five channels (F3, F4, C3, Cz and T7) on the frontal, central, and left temporal regions of the scalp produced an average AEP-age that was significantly correlated with chronological age $(p \leq 0.04)$. This refined AEP-age explained $17 \%$ of the variance in chronological age $F$ $(1,65)=13.42, p=0.001, \eta^{2}=0.24$ (see Figure 3 ) and was significantly different across chronological age groups, $F(3,66)=6.61, p<0.001$. Post-hoc analysis revealed that this refined AEP-age differentiated maturation between the younger (7 -8 year) and older (9-10 year) age groups (see Figure 4). 
Table 1

Comparison of Average AEP-Age, Oral Language Ability and Nonverbal IQ by Chronological Age Group

\begin{tabular}{lcccc}
\hline \multicolumn{1}{c}{ Group } & 7 years & 8 years & 9 years & 10 years \\
& $(n=15)$ & $(n=16)$ & $(n=22)$ & $(n=14)$ \\
\hline Mean age in years $(S D)$ & 7.58 & 8.51 & 9.41 & 10.35 \\
M:F ratio & $(.28)$ & $(.30)$ & $(.33)$ & $(.19)$ \\
AEP-age in years from 9 channels $(S D)$ & $9: 6$ & $7: 9$ & $9: 13$ & $6: 8$ \\
& $(.34)$ & $(.41)$ & $(.44)$ & 8.25 \\
AEP-age in years from 5 channels $(S D)$ & 7.95 & 7.83 & 8.52 & 8.46 \\
CELF-4 Language $(S D)$ & $(.42)$ & $(.60)$ & $(.60)$ & $(.60)$ \\
WASI PIQ $(S D)$ & 107 & 103 & 104 & 103 \\
& $(9)$ & $(9)$ & $(10)$ & $(12)$ \\
& 109 & 105 & 110 & 112
\end{tabular}

Note. CELF-4 and WASI are standard scores with $M=100$ and $S D=15$. 

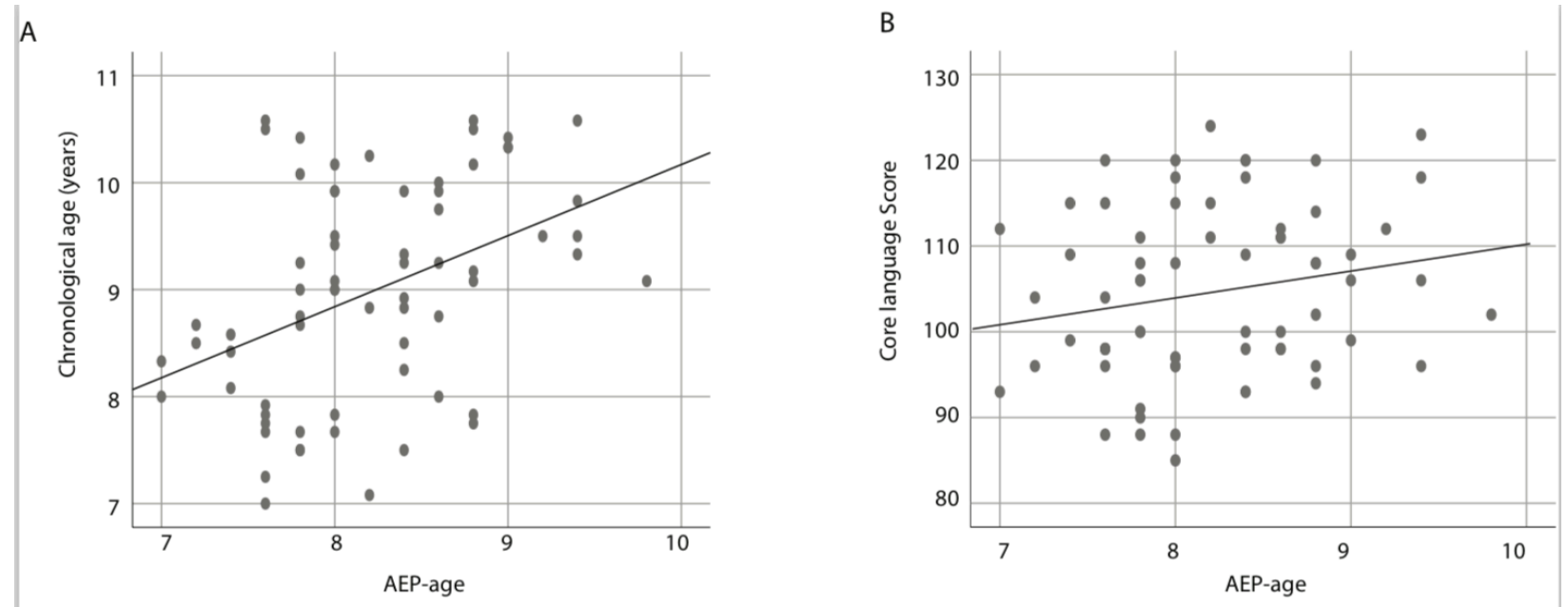

Figure 3. AEP-age derived from the five selected channels significantly predicted variance in chronological age (A) and core language score (B).

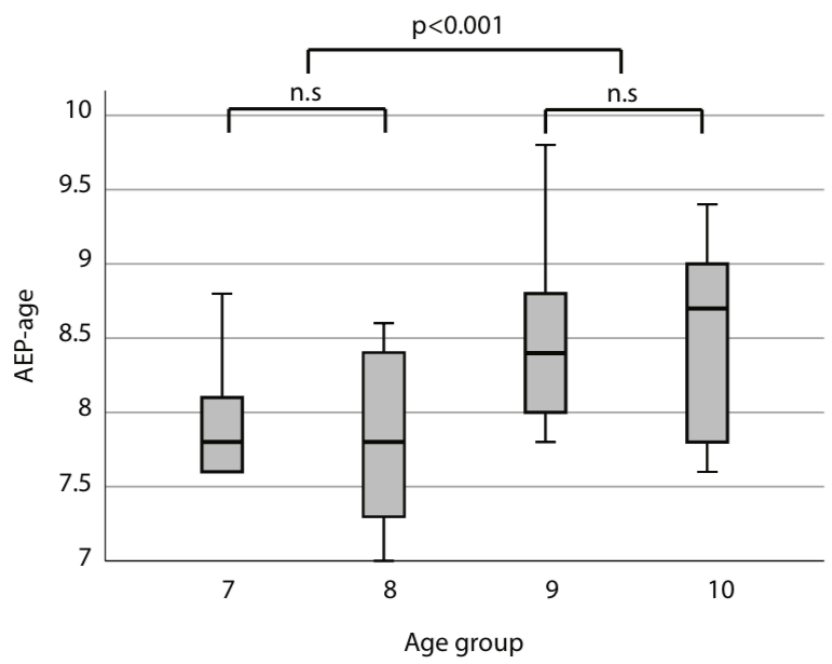

Figure 4. AEP-age did not differ between 7 and 8 year olds or between 9 and 10 year olds. AEP-age of 9 and 10 year olds was significantly higher than that of 7 and 8 year olds, $p<0.001$.

Hierarchical regressions explored the ability of AEP-age to predict language and nonverbal IQ (see Table 2). For language ability, the addition of refined AEP-age resulted in a significant $R^{2}$ change in the regression model, $F(2,65)=3.34, p=0.042$. AEP-age explained 
$7.8 \%$ of the variance in language ability beyond what was predicted by chronological age. By contrast, Performance IQ was not predicted by either chronological age or refined AEP-age. No significant model was found when AEP-age derived from the nine channels was entered into a hierarchical regression to predict language and nonverbal IQ $(F(2,65)=2.253, p=0.138$, $F(2,65)=0.063, p=0.802$ respectively $)$.

Table 2

Predicting Language Ability and Nonverbal IQ using Chronological Age and AEP-age

\begin{tabular}{|c|c|c|c|c|c|c|c|}
\hline & $t$ & $p$ & $\beta$ & $F$ & $d f$ & $\operatorname{Adj} R^{2}$ & $\Delta R^{2}$ \\
\hline \multicolumn{8}{|l|}{ Language ability } \\
\hline Step 1 & & & & 1.118 & 1,66 & 0.002 & 0.017 \\
\hline Chronological age & -1.058 & 0.294 & -0.257 & & & & \\
\hline Step 2 & & & & 3.343 & 2,65 & 0.066 & $0.078 *$ \\
\hline Chronological age & -1.965 & 0.054 & -0.257 & & & & \\
\hline AEP-age & 2.343 & 0.022 & 0.306 & & & & \\
\hline \multicolumn{8}{|l|}{ Nonverbal IQ } \\
\hline Step 1 & & & & 0.175 & 1,66 & -0.013 & 0.003 \\
\hline Chronological age & -0.418 & 0.678 & -0.052 & & & & \\
\hline Step 2 & & & & 0.093 & 2,65 & -0.028 & 0.000 \\
\hline Chronological age & -0.626 & 0.745 & -0.045 & & & & \\
\hline AEP-age & -0.123 & 0.903 & -0.017 & & & & \\
\hline
\end{tabular}

Note: $* p<0.05$ (two-tailed) 


\section{Discussion}

This study examined the developmental maturity of cortical AEPs elicited by a simple, single tone using the ICC analytic approach. First, we confirmed that the ICC-derived AEP-age estimate is a reliable method for capturing developmental changes in AEPs in school-aged children: AEP-age differentiated children with typical development who were 7 and 8 years of age from those who were 9 and 10 years. Second, we determined that these developmental changes in AEPs were best represented at frontal, central, and left temporal channels. Third, we demonstrated that AEP-age predicted significant variance in language ability, but not nonverbal IQ. Children who had the strongest language skills relative to their same-aged peers showed the most mature auditory cortical responses.

In this cross-sectional sample of children, we confirmed that ICC-derived AEP-age is a sensitive approach for differentiating developmental changes in auditory brain responses from 78 years to 9-10 years. This finding is similar to that of Bishop et al. (2011), who demonstrated sensitivity of this index to changes from 7 to 9 to 11 years of age. One interpretation of the lack of difference between our 7- and 8-, and 9- and 10-year-old groups is that the ICC procedure is only sensitive to at least two years of developmental change in AEP waveforms. However, several important experimental limitations are worth considering. First, the distribution of ages in the current sample affected AEP-age estimation in some of our age bands. The AEP-age for 7 year olds was likely inflated and that of 10 year olds likely underestimated as a consequence of the ICC procedure: it was not possible for a child aged 7 to be assigned an AEP-age lower than 7 without a younger grand average waveform available for comparison (Bishop et al., 2011), with the same issue applying to calculation of AEP-age in the 10 year age band. Children aged 8 and 9 years did, however, have both younger and older grand averages available for comparison. Thus, these estimated AEP-age equivalents were most accurate, and were also significantly different, indicating adequate resolution of this ICC procedure at the one-year level. It is 
therefore likely that AEP-age could be used to capture year-to-year developmental changes in the four age groups examined here with enough children of younger ( 5 and 6 years) and older (11 and 12 years) ages available for comparison.

Our finding that chronological age was a significant predictor of AEP-age provides a conceptual replication of Bishop et al. (2011). However, chronological age predicted a lower portion of AEP-age variance in our study (one-fifth) compared to Bishop et al. (one-third). Differences between studies in both age distribution and study design may account for this finding. First, the two groups in Bishop et al. differed in age by two years, whereas the present study involved one-year age increments. It is possible that very little maturational change occurs between 7 and 8 years, or after age 10 until adolescence. The latter interpretation has been put forward by Ponton et al. (2000), who found that AEP morphology in children does not begin to resemble that of adults until approximately age 12. It is also possible that too few participants were included in the present study to detect potentially subtle differences in AEP-age. Second, Bishop et al. examined a cross-sequential sample. Our study involved a cross-sectional sample, which increases between-subject variance, and thus may have contributed to a reduction in variance explained by chronological age.

Another principal goal of our study was to determine whether AEP maturity as indexed using the ICC approach is related to cognitive abilities such as language and nonverbal IQ. AEPage was found to be a significant predictor of language ability beyond what was explained by chronological age, but neither chronological age nor AEP-age were significant predictors of performance IQ. This finding is consistent with prior studies linking AEP component amplitude or latency with later language and reading abilities (Benasich et al., 2006; Espy et al., 2004; Molfese et al., 1999, 2000), and with our previous work using magnetoencephalography (MEG). In a series of regression analyses of auditory evoked fields in 45 children with typical development, autism spectrum disorder, or language disorder, we demonstrated that the peak 
latency of an early evoked response to a single tone predicted language ability but not nonverbal IQ (Oram Cardy et al., 2008). Together, our past and current findings suggest that the neural measures of auditory cortical processing used in our work are not generic indicators of children's global (verbal and nonverbal) cognitive function, but rather may be specifically tied to language function. What aspects of language function these might be would be an important question for future research in this area. In our MEG work, receptive language function was more closely tied to auditory cortical responses than expressive language ability. Further exploration is warranted of the ability of auditory cortical processing to predict specific aspects of language function such as receptive, expressive, phonological, semantic, and morphologic/syntactic elements.

Since null results must always be interpreted with caution, our finding that age-adjusted nonverbal IQ was not related to AEP-age warrants replication in particular. The WASI Performance IQ scale includes two subtests. Possibly, some skills that are not specifically linguistic do relate to AEP maturation but were either not measured directly by these subtests or were not included in the protocol (e.g., processing speed, perceptual closure).

Traditional ERP measurement approaches have been effective in showing significant differences between groups, but have been less effective in classifying or discriminating between individuals (Bishop et al., 2007). The use of ICC to generate an AEP-age estimate, as described here, is a promising approach to identifying and understanding individual differences in brain maturity in typically-developing and clinical populations. By comparing an individual child's ERP waveform to a series of grand average waveforms from a typically developing population, we may obtain an individual estimate of that child's auditory cortical maturation and determine whether, and to what extent, the child is delayed. This study has provided more support for the possibility of establishing normative developmental waveforms for different ages that could facilitate future clinical applications. To this end, we are exploring the utility of the AEP-age 
index (in isolation and in concert with other ERP indices) for identifying language impairment in a larger sample, in clinical populations, and in younger children in our ongoing and future work.

\section{Acknowledgements}

This study was supported by Natural Sciences and Engineering Research Council

Discovery Grants 418406-2012 to J.C. and 371201-2009 to L.A. The authors would like to acknowledge Jessi Boehm, Charity McCarthy, Jillian Spratt, and Jackson Wilson for their assistance in data collection.

\section{Conflict of Interest Statement}

The authors have no conflict of interest to declare.

\section{Author Contributions}

J.C., M.J., and L.A. conceived the study and designed the methodology. E.K., M.S., and H.M. performed the study. E.K. performed the data analyses and wrote the article with input from all authors.

\section{Data Accessibility Statement}

The data used in this research were collected under provision of informed consent of the participants. Access to the data will be granted in line with that consent, subject to approval by the university ethics board and under a formal Data Sharing Agreement. The $490 \mathrm{~Hz}$ tone wav file and the Matlab code for ICC calculation will be available at figshare.com.

\section{Abbreviations.}

AEP Auditory event-related potential

CELF-4 Clinical Evaluation of Language Fundamentals, $4^{\text {th }}$ edition

CLS Core Language Score (standard score)

EEG Electroencephalography 
ERP Event-related potential

ICC Intraclass correlation

PIQ Performance IQ

WASI Wechsler Abbreviated Intelligence Scale 


\section{References}

Archibald, L.M.D., Oram Cardy, J., Joanisse, M. \& Ansari, D. (2013) Language, reading and math learning profiles in a school age epidemiological sample. PLOS ONE, 8, e77463.

Benasich, A.A., Choudhury, N., Friedman, J.T., Realpe-Bonilla, T., Chojnowska, C., \& Gou, Z. (2006) The infant as a prelinguistic model for language learning impairments: Predicting from event-related potentials to behavior. Neuropsychologia, 44, 396-411.

Bender, S., Oelkers-Ax, R., Resch, F. \& Weisbrod, M. (2006) Frontal lobe involvement in the processing of meaningful auditory stimuli develops during childhood and adolescence. Neuroimage, 33, 759-773.

Bishop, D.V.M., Anderson, M., Reid, C. \& Fox, A.M. (2011) Auditory development between 7 and 11 years: An event-related potential (ERP) study. PloS One, 6, e18993.

Bishop, D.V.M., Hardiman, M., Uwer, R. \& von Suchodoletz, W. (2007) Maturation of the longlatency auditory ERP: step function changes at start and end of adolescence. Developmental Sci., 10, 565-575.

Bishop, D.V.M. \& McArthur, G.M. (2005) Individual differences in auditory processing in specific language impairment: a follow-up study using event-related potentials and behavioural thresholds. Cortex, 41, 327-341.

Boersma, P. \& Weenink, D. (2011) Praat: doing phonetics by computer [Computer program]. Version 5.2.35.

Čeponiene, R., Cheour, M., \& Näätänen, R. (1998) Interstimulus interval and auditory eventrelated potentials in children: Evidence for multiple generators. Electroen. Clin. Neuro., $108,345-354$.

Edgar, J.C., Lanza, M.R., Daina, A.B., Monroe, J.F., Khan, S.Y., Blaskey, L., Cannon, K.M., Jenkins III, J., Qasmieh, S., Levy, S.E. \& Roberts, T.P. (2014) Missing and delayed 
auditory responses in young and older children with autism spectrum disorders. Front Hum Neurosci, 8, 417.

Espy, K.A., Molfese, D.L., Molfese, V.I. \& Modglin, A. (2004) Development of auditory eventrelated potentials in young children and relations to word-level reading abilities at age 8 years. Ann. Dyslexia, $\mathbf{5 4}, 9$.

Fox, A.M., Anderson, M., Reid, C., Smith, T. \& Bishop, D.V.M (2010) Maturation of auditory temporal integration and inhibition assessed with event-related potentials (ERPs). BMC Neurosc., 11, 49.

McArthur, G.M. \& Bishop, D.V.M. (2004) Which people with specific language impairment have auditory processing deficits? Cogn. Neuropsychol., 21, 79-94.

Molfese, D.L. (2000) Predicting dyslexia at 8 years of age using neonatal brain responses. Brain Lang., 72, 238-245.

Molfese, D.L., Molfese, V.J., \& Espy, K.A. (1999) The predictive use of event-relayed potentials in language development and the treatment of language disorders. Dev. Neuropsychol., 16, 415-431.

Oram Cardy, J.E., Flagg, E.J., Roberts, W. \& Roberts, T.P.L. (2008) Auditory evoked fields predict language ability and impairment in children. Int. J. Psychophysiol., 68, 170-175.

Pang, E.W. \& Taylor, M.J. (2000) Tracking the development of the N1 from age 3 to adulthood: an examination of speech and non-speech stimuli. Clin. Neurophysiol., 111, 388-397.

Ponton, C., Eggermont, J.J., Khosla, D., Kwong, B. \& Don, M. (2002) Maturation of human central auditory system activity: separating auditory evoked potentials by dipole source modeling. Clin. Neurophysiol., 113, 407-420.

Ponton, C. W., Eggermont, J.J., Kwong, B. \& Don, M. (2000) Maturation of human central auditory system activity: evidence from multi-channel evoked potentials. Clin. Neurophysiol., 111, 220-236. 
Shafer, V.L., Schwartz, R.G. \& Martin, B. (2011) Evidence of deficient central speech processing in children with specific language impairment: The T-complex. Clin. Neurophysiol., 122, 1137-1155.

Semel, E., Wiig, E.H. \& Secord, W. (2003) Clinical Evaluation of Language Fundamentals - 4th edition. The Psychological Corporation, New York.

Sussman, E., Steinschneider, M., Gumenyuk, V., Grushko, J. \& Lawson, K. (2008) The maturation of human evoked brain potentials to sounds presented at different stimulus rates. Hearing Res., 236, 61-79.

Wechsler, D. (1999) Wechsler Abbreviated Scale of Intelligence. The Psychological Corporation, Toronto 